INVESTIGACIONES

\title{
VARIABLES ASOCIADAS A LA GESTION ESCOLAR COMO FACTORES DE CALIDAD EDUCATIVA
}

\author{
Associated variables for the management school education quality as factors
}

\section{Pablo López}

Universidad de Chile, Facultad de Ciencias Sociales, Departamento de Educación. Teléfono (02) 9787732, dirección electrónica pablopez@uchile.cl

\section{Resumen}

La necesidad de mejorar la calidad de la educación que los niños y jóvenes de nuestro país están recibiendo está en el centro de la discusión, más aún si se considera que en la última década los recursos aportados al sector se han prácticamente cuadruplicado. Según la literatura especializada, además de los recursos se necesita una gestión escolar eficiente que permita instalar capacidades en la escuela que aseguren un buen desempeño de los y las estudiantes en evaluaciones de pruebas estandarizadas nacionales tales como: la prueba SIMCE, la PSU, y en internacionales como PISA y TIMSS.

En el presente trabajo se propone un modelo causal que identifica algunas variables que inciden en la gestión de las organizaciones escolares y las relaciones entre dichas variables, así como el efecto que la gestión tiene en la calidad de la educación de estas organizaciones.

Palabras clave: gestión escolar, modelo causal, calidad educativa.

\begin{abstract}
The need to improve the quality of education that children and youth of our country are receiving is in the center of the discussion, even if one considers that in the last decade contributed to the resources sector have almost quadrupled. According to the literatura specialized, in addition to the resources needed to enable efficient management school installed capacity at the school to ensure a good performance of the students in national assessments such as standardized tests: the test SIMCE, the PSU and such as PISA and TIMSS.

In this paper a causal model proposed that identifies some variables that affect the management of school organizations and the relationships between these variables and the effect that the management analysis is the quality of education of these organizations.

Key words: management school, causal model, educational quality.
\end{abstract}




\section{ANTECEDENTES TEORICOS Y EMPIRICOS}

En la última década en Chile se ha realizado una importante inversión de recursos en el sistema escolar; sin embargo, los resultados de los procesos educativos, medidos a través de diversos instrumentos de evaluación como la Prueba de Selección Universitaria (PSU) y el Sistema de Medición de la Calidad de la Educación (SIMCE), dan cuenta de que los niveles alcanzados distan de lo ideal, más aún, resultan altamente insatisfactorios tanto al final del proceso de Enseñanza Media como en los niveles intermedios.

Este escenario llama a profundizar en las causas que subyacen a estos resultados, haciendo surgir un conjunto de interrogantes que han orientado la investigación que se presenta en este estudio: ¿Cuál es la realidad organizacional de los centros educativos?, es decir ¿cuál es la estructura que éstos se han dado para responder a las demandas del medio y cómo se genera el entramado social entre sus actores? Ambos son factores esenciales al momento de conocer las razones por las cuales se obtienen dichos resultados.

Para lograr que los aprendizajes sean de calidad, se debe contar con una clara y eficiente gestión escolar al interior de los establecimientos educacionales. Un análisis preliminar nos indica que al menos existen tres factores que están afectando negativamente la gestión de las organizaciones educativas -e influyendo en sus resultados-: En primer lugar, la ausencia de un liderazgo efectivo de quienes dirigen estas organizaciones. En segundo lugar, un desconocimiento de herramientas modernas de gestión, "Diversos estudios revelan que son más eficaces las escuelas en las cuales existe una buena relación entre profesor y alumno y hay orden y claridad hacia las metas; también, en aquellas escuelas donde los directores organizan espacios de reflexión; establecen relaciones positivas con sus profesores; promueven la participación en las decisiones académicas e intercambios de experiencias e involucran a directivos, profesores, estudiantes y padres en el mejoramiento de los resultados" (Martinic, 2002: 27).

Un tercer factor es una cultura organizacional fuertemente arraigada y opuesta a los cambios organizacionales y que genera una resistencia de la cual se conocen y atacan sus caras y no sus verdaderas causas. Una de las manifestaciones más claras de este escenario se expresa en la escasa utilización de instrumentos orientados a evaluar la gestión de las organizaciones educativas, "Pese a que la evaluación ha sido central en el proceso de Enseñanza - Aprendizaje (los alumnos se evalúan y califican periódicamente en cuanto a su aprendizaje cognitivo, conducta, responsabilidad, destreza deportiva, artística y otras dimensiones), la cultura evaluativa en nuestro medio es débil, en el sentido que ha estado centrada en el alumno y no en otros aspectos de la acción educativa" (Raczynski, 2001: 10).

La gestión escolar es un elemento determinante de la calidad del desempeño de las escuelas, sobre todo en la medida que se incrementa la descentralización de los procesos de decisión en los sistemas educacionales (Alvariño, et al., 2004). En la actualidad, se releva la importancia de una buena gestión para el éxito de los establecimientos educacionales, con su impacto en el clima de la escuela, en la planificación, en las formas de liderazgo, en la optimización de los recursos y del tiempo, la eficiencia y por ende en la calidad de los procesos.

Brunner y Elacqua (2003) identifican un conjunto de factores que inciden en una educación efectiva, variables que a nivel de escuela tienen la mayor probabilidad de producir buenos resultados académicos, según revela la evidencia empírica acumulada 
internacionalmente, entre ellos: Liderazgo y cooperación; clima de aprendizaje; monitoreo continuo del progreso de los alumnos; evaluación frecuente del desempeño de sus profesores; profesores reconocidos por su desempeño y gestión autónoma.

J. Weinstein (2002) ha señalado la existencia de algunos factores críticos de una gestión escolar de calidad que se podrían asociar a mejores resultados pedagógicos, entre los que menciona están una escuela con sentido compartido de misión; liderazgo del director o equipo directivo legitimado por la comunidad escolar; trabajo en equipo entre directivos y docentes y un buen nivel de compromiso con los profesores; proceso de planificación institucional participativo; participación efectiva de los distintos actores del sistema educativo; clima laboral y de convivencia positiva entre docentes, entre directivos y profesores y entre profesores y alumnos; y una adecuada inserción del establecimiento con su entorno.

Si analizamos la corriente de investigación sobre eficacia escolar efectuada por (Purkey y Smith, 1985; Good y Brophy, 1986; Scheerens, Vermeulen y Pelgrum, 1989; Brandt, 1992; Creemers y Scheerens, 1994; Reynolds, Bollen, Creemers, Hopkins, Stoll y Lagerweij, 1997), podemos advertir que estas investigaciones han señalado que existen distintos tipos de variables que inciden en los rendimientos académicos, como las variables de origen, donde se encuentran las asociadas a la familia y a la comunidad, y las variables asociadas a la gestión de la escuela. Estas últimas serían aquellas que entregarían valor agregado, es decir, harían una diferencia en el aprendizaje de los alumnos, más allá de las variables que se encuentran fuera de la escuela. Las variables de la escuela se agrupan en las características de la organización escolar, por una parte, y los procesos de instrucción en el aula, por otra. En relación a las características de la organización escolar, destacan las metas y el sentido de misión compartidas, el liderazgo educativo y pedagógico, orientación general hacia los aprendizajes, clima organizacional, aprendizaje organizativo y desarrollo profesional; y participación e implicación de la comunidad educativa.

Para avanzar en el mejoramiento de la gestión escolar, a partir del fortalecimiento de su evaluación, resulta útil la adaptación de los modelos de calidad existentes en el mundo, los cuales han sido de gran importancia en el desarrollo de la calidad de la gestión en las organizaciones empresariales. Al respecto, en la literatura de investigación se encuentra entre los más conocidos el modelo japonés llamado Deming (1951), que fija diez criterios de calidad; el modelo americano Baldrige (1987), que contempla siete criterios, y el modelo Europeo de Calidad EFQM (1998), que contempla siete criterios, agrupados en agentes y resultados. Todos los modelos sin excepción consideran entre sus elementos fundamentales al liderazgo, la planificación, los recursos y los resultados. En los últimos años en la realidad chilena, también se han venido incorporando los aportes de modelos de calidad; sin embargo, los modelos de carácter empresarial no han logrado penetrar y más bien han servido de referencia para la creación de diseños propios, adaptados de manera específica al campo de la educación.

Uno de ellos es el desarrollado por el Ministerio de Educación de Chile denominado SACGE (Sistema de Aseguramiento de la Calidad de la Gestión Escolar) conformado por cinco elementos de gestión: Liderazgo, Gestión curricular, Convivencia escolar y apoyo a los estudiantes, Recursos y Resultados (MINEDUC, 2005: 37). Este modelo se ha aplicado en las escuelas públicas del país y ha sido complementado por otro instrumento aportado por los equipos técnicos del Ministerio, denominado marco para la "Buena Dirección", 
que define cuatro ámbitos que son: Liderazgo, Gestión Curricular, Gestión de recursos y Gestión de clima y convivencia (MINEDUC, 2007: 11).

\section{METODOLOGIA DEL ESTUDIO}

Esta investigación se ha planteado el análisis de variables asociadas a la gestión escolar de los centros educativos por la relevancia que tienen en la calidad educativa. Para los estudios empíricos se realizó un análisis causal (Goldberger y Duncan, 1973) utilizando un modelo de ecuaciones estructurales (Joreskög y Sörbom, 1993).

El modelado con ecuaciones estructurales incluye dos submodelos o componentes (Cea, 2002):

i) El modelo estructural: describe relaciones causales entre variables latentes (exógenas y endógenas). Se define por la siguiente ecuación: $\mathrm{y}=\mathrm{B \eta}+\Gamma \xi+\zeta$.

ii) El modelo de medición: incluye las relaciones de las variables latentes (exógena y endógena) con sus indicadores(o variables empíricas), y se representa por las siguientes ecuaciones:

1. Modelo de medición para Y (variable observable dependiente o endógena): $\mathrm{Y}=\Lambda_{\mathrm{y}} \mathrm{y}+\varepsilon$.

2. Modelo de medición para $X$ (variable observable independiente o exógena): $\mathrm{X}=\Lambda_{\mathrm{x}} \xi+\delta$.

De acuerdo con las investigaciones empíricas previas se propuso el siguiente modelo (proceso-producto) considerando variables muy cercanas a las argumentaciones teóricas y a aquellas variables generadas como consecuencia de la interacción de los comportamientos docentes y estudiantes.

\section{Tabla 1}

Modelo proceso-producto

\begin{tabular}{|l|}
\hline \multicolumn{1}{|c|}{ Proceso } \\
\hline Liderazgo educativo \\
\hline Planificación y estrategia \\
\hline Gestión de recursos \\
\hline Procesos \\
\hline Gestión de personas \\
\hline Satisfacción \\
\hline Resultados \\
\hline
\end{tabular}

\begin{tabular}{|c|}
\hline Producto \\
\hline Calidad educativa \\
\hline
\end{tabular}

Elaboración propia. 
Las variables asociadas a la gestión escolar se recogieron a través de una encuesta, cuya validación empírica se realizó utilizando ecuaciones estructurales con variables latentes; las que fueron modeladas utilizando el software LISREL 8.5.

Las dimensiones y variables del instrumento se muestran en la tabla 2. Los descriptores de cada variable fueron extraídos desde el modelo Europeo de Calidad EFQM (1998), que contempla siete criterios, agrupados en agentes y resultados y del modelo desarrollado por el Ministerio de Educación (2005) denominado SACGE.

Tabla 2

Dimensiones y variables asociadas a la gestión escolar

\begin{tabular}{|c|c|c|c|}
\hline Dimensiones & Variables & Dimensiones & Variables \\
\hline Liderazgo & $\begin{array}{l}\text { Compromiso } \\
\text { Reconocimiento }\end{array}$ & Satisfacción & $\begin{array}{l}\text { Nivel de agrado } \\
\text { con la gestión y } \\
\text { funcionamiento } \\
\text { del establecimiento }\end{array}$ \\
\hline $\begin{array}{l}\text { Planificación y } \\
\text { estrategia }\end{array}$ & $\begin{array}{l}\text { Desarrollo de la } \\
\text { estrategia } \\
\text { Comunicación }\end{array}$ & Resultados & $\begin{array}{l}\text { Logros del establecimiento } \\
\text { en todos sus ámbitos de gestión }\end{array}$ \\
\hline $\begin{array}{l}\text { Gestión de } \\
\text { personas }\end{array}$ & $\begin{array}{l}\text { Competencias } \\
\text { Motivación }\end{array}$ & $\begin{array}{l}\text { Calidad } \\
\text { Educativa }\end{array}$ & $\begin{array}{l}\text { Expectativas } \\
\text { Percepciones de } \\
\text { los docentes en } \\
\text { los ámbitos de gestión }\end{array}$ \\
\hline Recursos & $\begin{array}{l}\text { Gestión de } \\
\text { recursos } \\
\text { Innovación }\end{array}$ & & \\
\hline Procesos & $\begin{array}{l}\text { Identificación de procesos } \\
\text { Seguimiento y control de procesos }\end{array}$ & & \\
\hline
\end{tabular}

Elaboración propia.

La muestra está compuesta por 70 establecimientos de las regiones V, VIII y Región Metropolitana. De ellos: 32 (45,7\%) municipales, 23 (32,8\%) particulares subvencionados y particulares pagados $15(21,4 \%)$. Los instrumentos fueron aplicados a una muestra de 1.470 profesores y directivos trabajando con una base de 60.000 datos.

Procedimientos. La primera tarea consistió en desarrollar los análisis estadísticos descriptivos y en examinar la agrupación factorial que poseen los indicadores del instrumento de medida con fines confirmatorios.

En la segunda etapa se realizaron los análisis factoriales confirmatorios de ambos modelos de medición, tanto de variables latentes exógenas como endógenas definidas en el modelo. 
En la tercera etapa se aplicaron los sistemas de ecuaciones estructurales en la validación del modelo causal, en conjunto con el modelo estructural y de medición. A partir de los datos obtenidos mediante esta técnica se realizaron simultáneamente dos tipos de inferencias: i) inferencias estructurales, en los posibles nexos causales entre las variables definidas en el modelo y ii) inferencias de medida de las variables. Los resultados alcanzados nos permitieron determinar el grado de explicación que representa la capacidad de gestión sobre la calidad educativa.

\section{RESULTADOS}

a) Estudio 1: análisis empíricos de los modelos de medición y estructural, tanto para las variables latentes exógenas como endógenas especificadas en el modelo causal. Tal como se muestra en el diagrama: figura 1.

\section{Figura 1}

\section{Modelo Inicial}

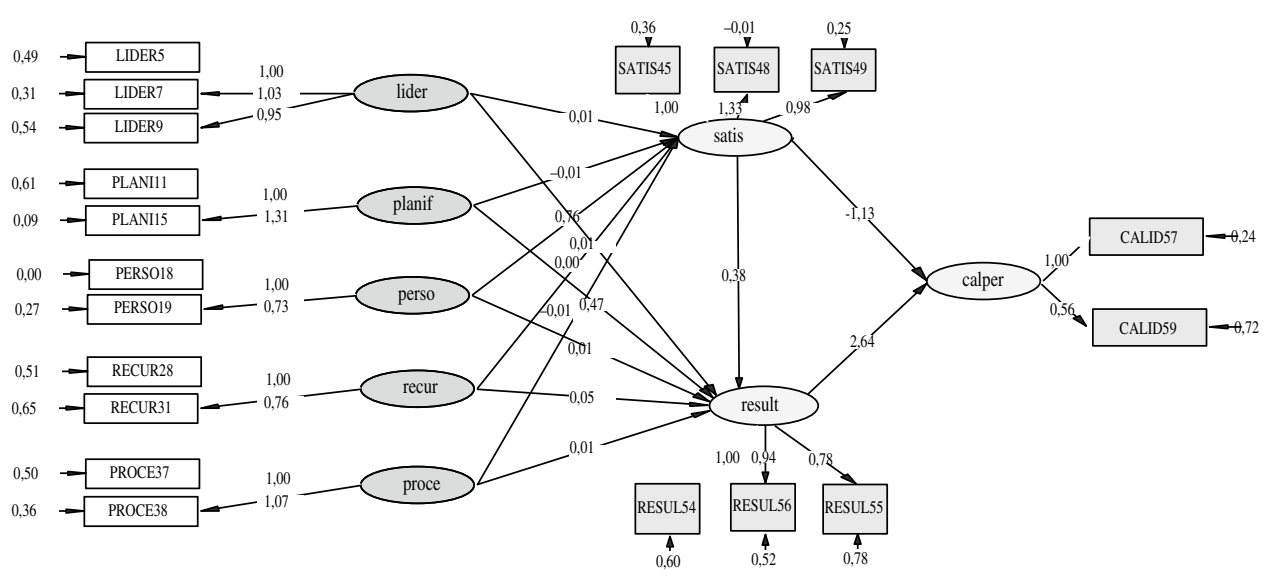

Chi-Square $=2091,45, \mathrm{df}=129, \mathrm{P}$-value $=0,00000$, RMSEA $=0,153$

En un primer análisis, se observa que la información que nos entrega el programa LISREL en relación a los coeficientes estimados en los modelos de medida y estructural exceden los límites aceptables. Por ejemplo, muestran varianza de error negativa, varianzas de error no significativa para los constructos y coeficientes estandarizados que sobrepasan el valor aceptable de 1.0,

Seguidamente, se presenta un conjunto de indicadores de bondad de ajuste del modelo inicial para el diagnóstico global. 
1. Normal Theory Weighted Least Squares Chi- Square: El estadístico $\chi^{2}$ del modelo modificado nos indica que el modelo no es aceptable, ya que los niveles de significación estadística no son mayores que 0,05 o 0,01 , por lo tanto las matrices de entrada previstas y efectivas son diferentes $\mathrm{Su}$ valor es de 2.091,45 con ( $\mathrm{p}=0,000)$.

2. Goodness of fit index (GFI): Indice de bondad de ajuste, su valor es de 0,75 , muy inferior al mínimo aceptable.

3. 90 Percent Confidence Interval for RMSEA: Intervalo de confianza para el error cuadrático medio de aproximación cuyo extremo inferior 0,16 , superior a 0,05 conduce a rechazar un error de marginalidad de la hipótesis para un ajuste aceptable.

4. Non-Normed Fit Index (NNFI): índice de ajuste no normado de Tucker y Lewis (1973). Su valor de 0,74, inferior a 0,95, indica un mal ajuste.

5. Comparative Fit Index (CFI): índice de ajuste comparado de Bentler (1990). Su valor de 0,80 , inferior a 0,95 , indica un muy mal ajuste.

6. $\chi^{2}$ normado: Jöreskog, propuso que la Chi-Cuadrado se ajuste por los grados de libertad para evaluar el ajuste en varios modelos. Su valor en este análisis es 16,2, lo que se puede interpretar como un ajuste de muy baja significación.

Se observa que los indicadores para el ajuste global del modelo nos muestran ajustes deficientes y por lo tanto habrá que reespecificar el modelo

b) Estudio 2: análisis causal del modelo final (figura 2). Este estudio se inició con el análisis de las saturaciones que presenta LISREL en relación al modelo, para continuar con el estudio de los índices de bondad de ajuste del modelo para el diagnóstico global.

Figura 2

Modelo Final reespecificado

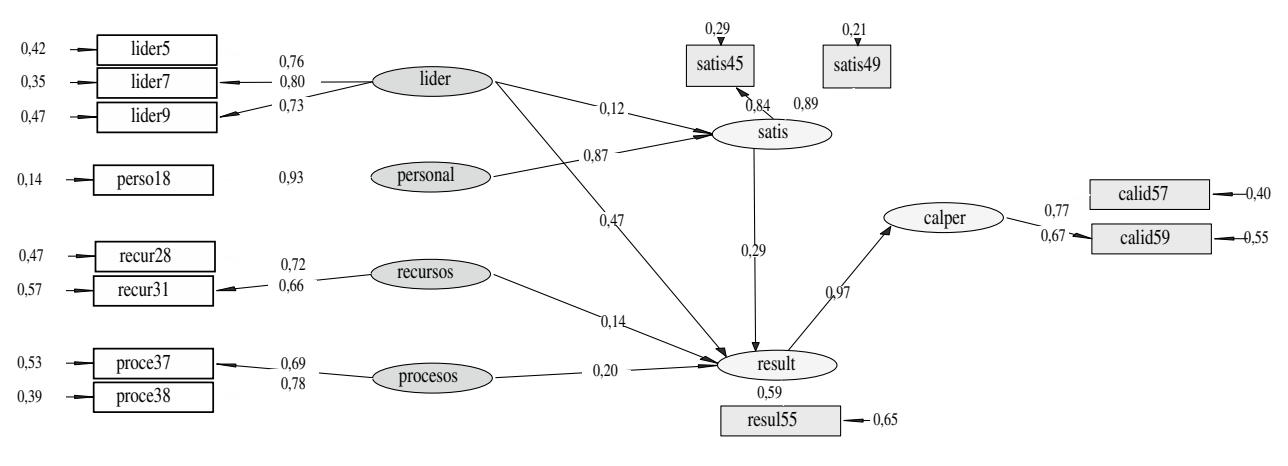

Chi-Square $=71,56, \mathrm{df}=49$, P-value $=0,01942$, RMSEA $=0,027$

Las ecuaciones que se presentan y que articulan las variables latentes exógenas (V.I) con las variables endógenas (V.D) presentan valores aceptables, no se presentan problemas de varianzas de error negativas o errores estándar muy elevados asociados a coeficientes 
estimados, lo que nos indica, en base a la comparación con varios modelos que se han estimado, que finalmente éste será el modelo definitivo.

\section{Figura 3}

Ecuaciones del modelo, con las estimaciones de sus coeficientes de regresión, saturaciones, varianzas de error, varianzas de perturbación, y coeficientes de determinación

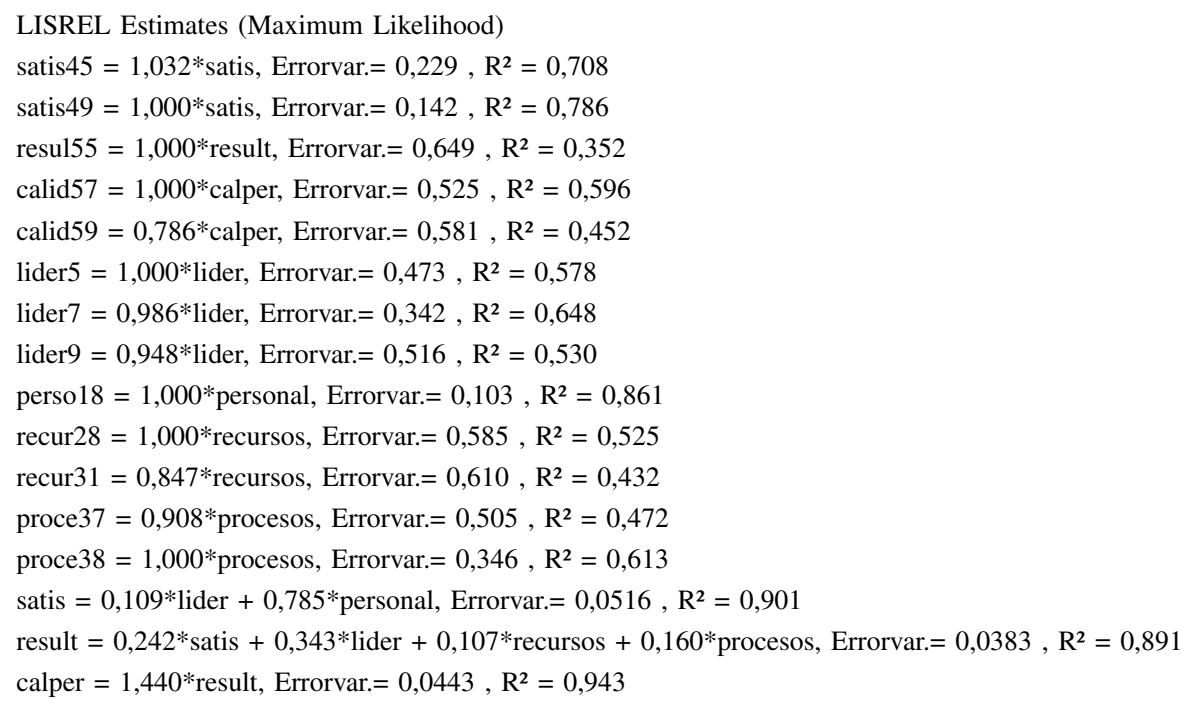

\section{Indices de bondad de ajuste del modelo final para el diagnóstico global}

Este análisis nos permitirá descubrir el grado de ajuste entre el modelo y los datos una vez que se ha puesto a prueba. Este conjunto de indicadores de ajuste sirve para la interpretación de los resultados. Los tres tipos de medidas de ajuste, utilizadas en el análisis, pueden representarse por las medidas de ajuste absoluto, incremental y de parsimonia. En este estudio nos centraremos en un número limitado de cada tipo de medida.

1. Normal Theory Weighted Least Squares Chi-Square (ajuste absoluto): El estadístico $\chi^{2}$ del modelo modificado nos indica que el modelo es marginal pero aceptable. Su valor de 93.490 con $(\mathrm{p}=0,000133)$.

2. Goodness of fit index (GFI) (ajuste absoluto): Indice de bondad de ajuste, su valor es de 0,978 , valor superior al mínimo aceptable, aun cuando no se ha establecido ningún umbral de aceptabilidad.

3. 90 Percent Confidence Interval for RMSEA (ajuste absoluto): Intervalo de confianza para el error cuadrático medio de aproximación, cuyo extremo inferior 0,0110 , inferior a 0,05 conduce a aceptar la hipótesis.

4. Non-Normed Fit Index (NNFI) (ajuste incremetal): Indice de ajuste no normado de Tucker y Lewis (1973). Su valor de 0,982, valor superior a 0,95, indica un buen ajuste. 
5. Comparative Fit Index (CFI) (ajuste incremental): Indice de juste comparado de Bentler (1990). Su valor de 0,989, superior a 0,95, indica un buen ajuste.

6. $\chi^{2}$ normado (ajuste de parsimonia): Jöreskog, propuso que la Chi-Cuadrado se ajuste por los grados de libertad para evaluar el ajuste en varios modelos. Su valor es igual a 1,91 , lo que se puede interpretar como ajuste aceptable.

Los modelos de ecuaciones estructurales también incluyen los índices que pueden dar luz a los procesos de modificación del modelo: los índices de modificación para aplicar el contraste de los multiplicadores de Lagrange sobre los parámetros ausentes del modelo, junto con el valor aproximado de sus estimaciones. LISREL sólo muestra una selección de los parámetros más significativos (figura. 4).

\section{Figura 4}

Multiplicadores de Lagrange

The Modification Indices Suggest to Add the

Path to from Decrease in Chi-Square New Estimate

calid59 satis $\quad 9,1 \quad-0,29$

lider9 personal $\quad 13,2 \quad 0,26$

perso18 procesos $\quad 11,3 \quad-0,42$

\section{The Modification Indices Suggest to Add an Error Covariance}

Between and Decrease in Chi-Square New Estimate

\begin{tabular}{lrr} 
calper satis $11,5 \quad-0,12$ \\
\hline
\end{tabular}

recur31 resul55 $8,0 \quad 0,08$

En relación al diagrama, se confirma, de acuerdo a la teoría, el efecto directo de la satisfacción sobre los resultados y de los resultados sobre la calidad, además del efecto indirecto de la satisfacción sobre la calidad a través de los resultados, los cuales explican un porcentaje importante de la varianza.

Con esta reespecificación del modelo probamos en diferentes muestras la validez empírica del modelo de ecuaciones estructurales.

\section{Tabla 3}

Resumen comparativo de los índices de bondad de ajuste del modelo reespecificado

\begin{tabular}{|l|c|c|c|c|c|}
\hline $\begin{array}{c}\text { Indices de } \\
\text { Ajuste }\end{array}$ & $\begin{array}{c}\text { Muestra } \\
\text { Completa }\end{array}$ & $\begin{array}{c}\text { Submuestra } \\
\text { Aleatoria }\end{array}$ & $\begin{array}{c}\text { Submuestra } \\
\text { Colegios } \\
\text { Municipalizados }\end{array}$ & $\begin{array}{c}\text { Submuestra } \\
\text { Colegios } \\
\text { Particulares } \\
\text { Subvencionados }\end{array}$ & $\begin{array}{c}\text { Submuestra } \\
\text { Colegios } \\
\text { Particulares } \\
\text { Pagados }\end{array}$ \\
\hline CAIC & 385.914 & 342.741 & 399.574 & 376.138 & - \\
\hline$\chi^{2} / \mathrm{gl}$ & 1,46 & 1,13 & 1,8 & 1,47 & - \\
\hline CFI & 0,989 & 0,989 & 0,982 & 0,985 & - \\
\hline
\end{tabular}




\begin{tabular}{|l|c|c|c|c|c|}
\hline $\begin{array}{c}\text { Indices de } \\
\text { Ajuste }\end{array}$ & $\begin{array}{c}\text { Muestra } \\
\text { Completa }\end{array}$ & $\begin{array}{c}\text { Submuestra } \\
\text { Aleatoria }\end{array}$ & $\begin{array}{c}\text { Submuestra } \\
\text { Colegios } \\
\text { Municipalizados }\end{array}$ & $\begin{array}{c}\text { Submuestra } \\
\text { Colegios } \\
\text { Particulares } \\
\text { Subvencionados }\end{array}$ & $\begin{array}{c}\text { Submuestra } \\
\text { Colegios } \\
\text { Particulares } \\
\text { Pagados }\end{array}$ \\
\hline GFI & 0,978 & 0,969 & 0,972 & 0,972 & - \\
\hline AGFI & 0,960 & 0,942 & 0,948 & 0,948 & - \\
\hline RMR & 0,025 & 0,0301 & 0,0295 & 0,0269 & - \\
\hline REMSEA & 0,027 & 0,0202 & 0,0362 & 0,0306 & - \\
\hline
\end{tabular}

Al observar los índices de bondad de ajuste, tanto en la muestra completa, submuestras aleatorias, submuestras colegios particulares pagados, subvencionados y municipalizados éstos en general son similares. En muestras pequeñas los valores no logran ajustarse como en muestras significativas. En el caso relativo a la muestra tomada de los colegios particulares pagados, los valores que se obtuvieron no fueron satisfactorios.

En las muestras pequeñas menores a cien sujetos, como el caso de los colegios particulares donde se encuestaron setenta y siete profesores, número insuficiente aconsejado por planteamientos teóricos, el programa LISREL no logra pasar la fase de identificación, por lo que no se presentan indicadores.

\section{CONCLUSIONES}

De acuerdo a los datos y análisis realizados, se presentan las conclusiones de los resultados obtenidos por medio del estudio empírico.

Los indicadores relativos al Liderazgo, como "compromiso y reconocimiento", son valorados por los profesores, como fundamentales para el desarrollo de los establecimientos educativos.

La dimensión planificación y estrategia, asociada con las organizaciones escolares, a través de los indicadores "desarrollo y comunicación de las estrategias", es la dimensión menos valorada por los educadores, situándose muy por debajo de las valoraciones de las otras dimensiones.

La dimensión gestión de personas tiene una baja valoración, aun cuando se observa que el indicador "motivación" presenta una mejor valoración, que los indicadores relativos a "competencias".

En relación a la dimensión recursos, las valoraciones son positivas en torno a sus indicadores: "gestión de los recursos e innovación". Los profesores valoran contar con recursos tecnológicos y didácticos a su disposición, que les permitan realizar innovaciones de enseñanza.

Respecto de la dimensión procesos, la mejor valoración es la "identificación de procesos en materia de enseñanza y evaluación", mientras que el indicador "seguimiento y control de los procesos" presenta una baja valoración por parte de los profesores.

En relación a los efectos directos que muestra el modelo final, las variables que asocian las dimensiones de liderazgo, recursos y procesos, presentan un efecto estadístico significativo sobre la variable resultados. Los efectos de las dimensiones planificación, recursos y procesos no presentan efectos estadísticamente significativos sobre la variable 
satisfacción, sin embargo liderazgo y gestión de personal presentan efecto sobre la satisfacción, lo cual nos indica que existe una relación entre "motivación y reconocimiento" por parte de los directivos a los profesores en su labor docente y el compromiso que la organización debe asumir con su formación.

Acerca de la relación entre las variables endógenas presentadas en el modelo, se puede señalar que la dimensión satisfacción tiene efecto directo sobre la variable resultados, pero no sobre la calidad educativa, mientras que la dimensión resultados tiene efecto directo sobre la dimensión calidad percibida.

En cuanto a los efectos indirectos que se presentan en el modelo final, se observa que las dimensiones de liderazgo, recursos y procesos poseen efectos estadísticos significativos a través de los resultados sobre la calidad educativa. En relación a las demás variables sus efectos indirectos sobre la calidad educativa no son estadísticamente significativos.

En síntesis, éstas son las conductas más significativas de cada una de las dimensiones que se presentan en el modelo causal.

También se sometió a prueba el modelo reespecificado a diferentes submuestras, tanto por separado como a través del análisis multimuestra. Al respecto se pudo observar que los parámetros muestran cierta estabilidad cuando los tamaños de las muestras son elevados (mayores de 200 sujetos), mientras que en muestras pequeñas menores a 100 sujetos, los parámetros y los índices de ajuste manifiestan claramente un débil ajuste a los datos, por lo tanto nuestra hipótesis sobre la no variabilidad de los parámetros para diferentes grupos considerados simultáneamente, de acuerdo a ciertas variables de control, en rigor y globalmente no son confirmadas.

Actualmente, más que nunca en nuestro país, se requiere rigurosidad y sistematicidad en todo el quehacer orientado a mejorar la calidad de la educación. No es posible insistir en la aplicación de herramientas, que pueden ser modernas o que han resultado efectivas en otros países, sin someterlas a la validación empírica que un proceso que pretende ser científico necesariamente debe realizar.

\section{BIBLIOGRAFIA}

Alvariño, C., S. Arzola, J. J. Brunner, M. Recart y R. Vizcarra (2004). Gestión Escolar: Un estado del arte de la literatura, http:// www.usuarios.lycos.es/invgerprocesoedu/lecturas/gestion_htm.

Brunner, J. J. y G. Elacqua (2003). Factores que inciden en una educación efectiva, http://www. archivochile.com/edu.doc_analit/est_doc_analit00027.pdf.

Creemers, B. \& J. Scheerens (1994). Development in the educational effectiveness research program. En International Journal of Educational Research, 2 (21), pp. 125-139.

Good, T. \& J. Brophy (1986). School Effects. En M. C. Wittrock (ed.), Handbook of research on teaching, Nueva York, Mcmillan, pp. 656-687.

Martinic, S. (2002). Gestión efectiva para mejorar los aprendizajes, Revista de Educación, Ministerio de Educación $\mathrm{N}^{\mathrm{o}}$ 292, Chile, pp. 27-28.

Ministerio de Educación (2005). Calidad en todas las escuelas y liceos. Sistema de aseguramiento de la calidad de la gestión escolar, Santiago, Chile.

Ministerio de Educación (2007). Marco para la buena dirección. Santiago, Chile.

Purkey, S. \& M. Smith (1985). School reform: the distric policy implications of the effectives school literature. En Elementary School Journal, 85, pp. 353-389. 
Raczynski, D. (2001). Evaluación: una herramienta para mejorar, Ministerio de Educación, Revista de Educación, $\mathrm{N}^{\mathrm{o}}$ 290, Chile, pp. 9-12.

Reynolds, D., R. Bollen, B. Creemers, D. Hopkins, L. Stoll y N. Largerweij (1997). Las Escuelas Eficaces claves para mejorar la enseñanza, Editorial Santillana, Madrid.

Scheerens, J., A. Vermeulen \& W. Pelgrum (1989). Generability of instructional and school effectiveness indicators across nations. En Creemers y Scheerens (Editores), Special Issue of International Journal of Educational Research, 13 (7), pp. 789-800.

Weinstein, J. (2002). Calidad y Gestión: Condiciones y Desafíos, Revista Pensamiento Educativo. Vol. 31, pp. 50-71, Pontificia Universidad Católica de Chile.

\section{BIBLIOGRAFIA METODOLOGIA}

Cea, M. (2002). Análisis Multivariante. Teoría y práctica en la investigación Social, Editorial Síntesis, Madrid, pp. 538-582.

Goldberger and Duncan (1973). Structural Equation Models in the Social Sciencies, Editorial Academic Press, New York.

Joreskög, K. \& D. Sorböm (1993). LISREL 8, Structural Equation Modeling with the Simples Language, Editorial Scientific Sofware International, inc., USA.

Ministerio de Educación (2005). Calidad en todas las escuelas y liceos. Sistema de aseguramiento de la calidad de la gestión escolar, Santiago, Chile. 